\title{
Bone Subtraction lodine Imaging Using Area Detector CT for Evaluation of Skull Base Invasion by Nasopharyngeal Carcinoma
}

\author{
(D)T. Hiyama, (DH. Kuno, (D) K. Sekiya, (D) S. Tsushima, D O. Sakai, (D) M. Kusumoto, and (D)T. Kobayashi
} om E

\begin{abstract}
BACKGROUND AND PURPOSE: Conventional CT has generally lower detectability of bone marrow invasion than MR imaging due to lower tissue contrast. The purpose of this study was to compare the diagnostic performance of conventional CT alone or in combination with bone subtraction iodine imaging using area detector CT for the evaluation of skull base invasion in patients with nasopharyngeal carcinoma.
\end{abstract}

MATERIALS AND METHODS: Forty-four consecutive patients who underwent contrast-enhanced CT using 320-row area detector CT and contrast-enhanced MR imaging for nasopharyngeal carcinoma staging between April 2012 and November 2017 were enrolled in this retrospective study. Bone subtraction iodine images were generated by subtracting pre- and postcontrast volume scans using a highresolution deformable registration algorithm. Two blinded observers evaluated skull base invasion at multiple sites (sphenoid body, clivus, bilateral base of the pterygoid process, and petrous bone) using conventional CT images alone or in combination with bone subtraction iodine images. Examination of MR and CT images by an experienced neuroradiologist was the reference standard for evaluating sensitivity, specificity, and area under the receiver operating characteristic curve.

RESULTS: Twenty-six patients (59\%) showed skull base invasion at 84 sites on the reference standard. Conventional CT plus bone subtraction iodine images showed higher sensitivity $(92.9 \%$ versus $78.6 \%, P=.02)$ and specificity $(95.6 \%$ versus $86.1 \%, P=.01)$ than conventional CT images alone for evaluating skull base invasion. The area under the receiver operating characteristic curve for conventional CT plus bone subtraction iodine $(0.98)$ was significantly larger $(P<.001)$ than the area under the receiver operating characteristic curve for conventional CT alone (0.90).

CONCLUSIONS: Conventional CT plus bone subtraction iodine performs more closely to the accuracy of combining CT and MR imaging compared with conventional CT alone.

ABBREVIATIONS: $\mathrm{AUC}=$ area under the receiver operating characteristic curve; $\mathrm{BSI}=$ bone subtraction iodine; $\mathrm{CCT}=$ conventional $\mathrm{CT} ; \mathrm{NPC}=$ nasopharyngeal carcinoma

$\mathbf{N}$ asopharyngeal carcinoma (NPC), arising from the epithelial lining of the nasopharynx, has a propensity to invade the skull base just above the nasopharynx. Skull base invasion indicates a tumor stage of at least T3 according to the eighth edition of

Received June 20, 2018; accepted after revision October 22.

From the Department of Diagnostic Radiology (T.H., H.K., K.S., M.K., T.K.), National Cancer Center Hospital East, Kashiwa, Chiba, Japan; Canon Medical Systems Corporation (S.T.), Otawara, Tochigi, Japan; Departments of Radiology (O.S.), Otolaryngology-Head and Neck Surgery (O.S.), and Radiation Oncology (O.S.), Boston Medical Center, Boston University School of Medicine, Boston, Massachusetts; and Department of Diagnostic Radiology (M.K.), National Cancer Center Hospital, Chuo-ku, Tokyo, Japan.

This study was supported by a grant from the Japanese Ministry of Education, Culture, Sports, Science and Technology (Grant-in-Aid for Young Scientists [B] KAKEN; No. 26861033) and Canon Medical Systems.

Paper previously presented, in part, at Annual Meeting of the American Society of Neuroradiology and the Foundation of the ASNR Symposium, June 2-7, 2018; Vancouver, British Columbia, Canada. the American Joint Committee on Cancer staging system. ${ }^{1} \mathrm{NPC}$ is treated mainly by radiation therapy, with or without chemotherapy. Intensity-modulated radiation therapy has gradually become the standard treatment for NPC because of its superior tumor target conformity and significantly improved localization. ${ }^{2,3}$ As the spatial accuracy of dose distribution improves, a more precise evaluation of the spatial extent of NPC is required.

MR imaging offers excellent soft-tissue contrast and is useful for the diagnosis, staging, and evaluation of tumor extent. ${ }^{4-7}$ It is

Please address correspondence to Hirofumi Kuno, MD, PhD, Department of Diagnostic Radiology, National Cancer Center Hospital East, 6-5-one Kashiwanoha, Kashiwa, Chiba 277-8577, Japan; e-mail: hkuno@east.ncc.go.jp

-- Indicates open access to non-subscribers at www.ajnr.org

三 Indicates article with supplemental on-line appendix.

Indicates article with supplemental on-line photos.

http://dx.doi.org/10.3174/ajnr.A5906 
well-established that MR imaging is better than CT at identifying invasion of the skull base. ${ }^{8-11}$ CT can detect skull base involvement manifesting as lytic or sclerotic lesions but has lower sensitivity than MR imaging due to lower tissue contrast and lower detectability of bone marrow invasion. However, CT is often the primary imaging technique for radiation therapy planning and dose computation and may be the only option for patients with contraindications to MR imaging such as intracranial or orbital metallic foreign bodies and MR imaging-unsafe pacemaker devices, or for patients who cannot tolerate MR imaging because of claustrophobia.

Currently, digital subtraction angiography-like bone subtraction iodine (BSI) imaging with $160-\mathrm{mm}$-wide area detector CT is used in some musculoskeletal and neurologic applications. ${ }^{12-14}$ According to Gondim Teixeira et al, ${ }^{12}$ BSI imaging with area detector CT showed high performance for the visual identification of bone marrow enhancement adjacent to lytic bone lesions. This technique reduces spatial mismatch by using volume scanning with wide area detector CT and a high-resolution deformable registration algorithm, enabling identification of contrast enhancement in the bone marrow. We hypothesized that BSI imaging might be useful for detecting skull base invasion and accurately assessing the extent of bone invasion by the NPC.

Hence, the purpose of this retrospective study was to compare the diagnostic performance of conventional CT (CCT) alone and in combination with BSI imaging using area detector CT for the evaluation of skull base invasion in patients with NPC.

\section{MATERIALS AND METHODS Study Population}

Our institutional review board at National Cancer Center Hospital East approved this retrospective study. The requirement to obtain written informed consent was waived. Between April 2012 and November 2017, forty-seven consecutive newly diagnosed patients with histologically proved NPC underwent contrast-enhanced CT for cancer staging before treatment. Among them, 46 patients also underwent contrast-enhanced 3T MR imaging before treatment. Two of the 46 patients were excluded because of unavailable volume data needed for subtraction reconstruction. The remaining 44 patients ( 28 men, 16 women; age range, 18-79 years; mean age, 60 years) were enrolled in this study. Of the 44 patients, $29(65.9 \%)$ had nonkeratinizing squamous cell carcinoma and 8 (18.2\%) had keratinizing squamous cell carcinoma. The detailed clinical profiles are summarized in Table 1. Tumor stage was determined according to the seventh edition of the American Joint Committee on Cancer staging system. ${ }^{15}$

\section{Digital Subtraction Angiography-Like BSI Imaging Techniques}

All CT studies were performed using a 320 - detector row CT system with a detector width of $160 \mathrm{~mm}$ (Aquilion ONE Vision; Canon Medical Systems, Otawara, Japan), with the following parameters: $120 \mathrm{kV}, 120$ effective mAs (milliampere-second), 0.5second rotation time, $160-\mathrm{mm}$ collimation. The average CT dose index was $25.2 \mathrm{mGy}$. Patients received a $100-\mathrm{mL}$ injection of 300 $\mathrm{mg} \mathrm{I} / \mathrm{mL}$ of iodinated contrast medium (iopamidol; Teva Takeda Yakuhin, Koka, Japan) at a rate of $2.5 \mathrm{~mL} / \mathrm{s}$ into an antecubital
Table 1: Summary of patient characteristics

\begin{tabular}{|c|c|c|}
\hline Characteristics & No. & $\%$ \\
\hline \multicolumn{3}{|l|}{ Age (yr) } \\
\hline Mean & 60 & \\
\hline Range & 18-79 & \\
\hline \multicolumn{3}{|l|}{ Sex } \\
\hline Female & 16 & $36.0 \%$ \\
\hline Male & 28 & $64.0 \%$ \\
\hline \multicolumn{3}{|l|}{ Histopathology } \\
\hline Nonkeratinizing carcinoma & 29 & $65.9 \%$ \\
\hline Differentiated & 8 & \\
\hline Undifferentiated & 19 & \\
\hline Unknown & 2 & \\
\hline Keratinizing carcinoma & 8 & $18.2 \%$ \\
\hline Unknown & 7 & $15.9 \%$ \\
\hline \multicolumn{3}{|l|}{ TNM (7th AJCC) } \\
\hline $\mathrm{Tl}$ & 8 & $18.2 \%$ \\
\hline $\mathrm{T} 2$ & 10 & $22.7 \%$ \\
\hline T3 & 4 & $9.1 \%$ \\
\hline T4 & 22 & $50.0 \%$ \\
\hline NO & 3 & $6.8 \%$ \\
\hline $\mathrm{N} 1$ & 16 & $36.4 \%$ \\
\hline N2 & 13 & $29.5 \%$ \\
\hline $\mathrm{N} 3 \mathrm{a}$ & 1 & $2.3 \%$ \\
\hline N3b & 11 & $25.0 \%$ \\
\hline MO & 41 & $93.2 \%$ \\
\hline M1 & 3 & $6.8 \%$ \\
\hline \multicolumn{3}{|l|}{ Subsite } \\
\hline Posterior superior & 12 & $27.3 \%$ \\
\hline Lateral wall & 32 & $72.7 \%$ \\
\hline
\end{tabular}

Note:-TNM indicates tumor, node, metastasis tumor stage; AJCC, American Joint Committee on Cancer.

vein through a 22-ga cannula. Scans were started 7 (mask volume) and 70 seconds (postcontrast volume) after the start of the injection in an intermittent acquisition mode with no table feed. During volume scanning, the patient's head was fixed to the bed of the CT with a headband and neck collar (Stifneck Select; Laerdal, Stavanger, Norway) (On-line Fig 1).

Two volume datasets (7- and 70-second CT) were reconstructed using a high-resolution deformable registration algorithm. The mask volume was subtracted from the postcontrast volume using the ${ }^{\mathrm{SURE}}$ Subtraction application (Canon Medical Systems). The high-resolution deformable registration algorithm is described in detail in the On-line Appendix and On-line Fig 2. ${ }^{16}$ Axial and coronal CCT images with soft-tissue (window level 65, window width 330) and bone (window level 650, window width 3000 ) windows, and axial and coronal BSI images (window level 40 , window width 180) were reconstructed with a $2-\mathrm{mm}$ slice thickness, $512 \times 512$ matrix, and 16-cm FOV. The CCT and BSI images were reconstructed to yield precisely matching slices.

\section{Image Interpretation}

Two independent radiologists (with 12 years [H.K.] and 24 years [T.K.] of experience in oncologic diagnostic radiology), blinded to the patients' clinical histories and to the images from the other technique, independently analyzed the images. The CCT images and combined CCT-plus-BSI images were evaluated using a 5-point scale for calculating the area under the receiver operating characteristic curve (AUC) and weighted $\kappa$ statistics. For CCT images, findings for skull base invasion were considered negative when the tumor was separated from the skull base (score 1, defi- 
nitely negative) or was only in contact with the skull base (score 2, probably negative). Findings of skull base invasion were considered present when erosion or sclerosis (score 3, possibly positive), erosion and sclerosis (score 4 , probably positive), or invasion into the bone marrow (score 5, definitely positive) was present. For combined analysis of CCT and BSI images, we scored skull base invasion using the BSI image in addition to the CCT image. If the BSI image showed definitive bone enhancement continuous with the tumor, we scored it as grade 5 (definitely positive); the absence of enhancement was scored as grade 2 (probably negative). If the bone marrow enhancement was equivocal or impossible to estimate, we gave scores of 2 , three (possibly positive), or 4 (probably positive) based on the confidence level of enhancement on BSI images and findings on CCT. By means of a workstation (Shade Quest View R; Yokogawa Electric, Tokyo, Japan), the images were presented in random order in 2 sessions, initially with CCT alone (soft-tissue and bone window), followed 4 weeks later by a combination of CCT and BSI images. The readers were able to generate fusion images of the bone window and BSI images. Skull base invasion was evaluated at 6 sites on the skull base (sphenoid body, clivus, bilateral pterygoid process, and bilateral petrous apex). The final score for the skull base invasion was determined by a consensus between the 2 readers.

\section{Standard of Reference}

Owing to the difficulty in obtaining histopathologic confirmation of skull base invasion, a combination of MR imaging and CT features observed by an experienced neuroradiologist (T.H., 12 years of experience in neuro-/head and neck radiology) who was not involved in image interpretation in this study but made reports clinically in some cases was used as the reference standard for evaluating sensitivity, specificity, and the AUC for skull base invasion. The MR imaging protocol is discussed in the On-line Appendix. All patients underwent MR imaging and CT examinations within a median of 5 days (range, $0-23$ days).

\section{MR Imaging and CT Diagnostic Criteria for Skull Base Invasion}

Findings of skull base invasion were considered positive in cases in which the skull base had signals similar to those of the adjacent tumor on all pre- and postcontrast T1-weighted images in the cortical bone and bone marrow space on the MR image (low signal intensity on a T1-weighted image and contrast enhancement on the postcontrast T1-weighted image with fat suppression). ${ }^{8,9,17-21}$ We performed evaluations mainly on the basis of the precontrast 3D T1-weighted Dixon in-phase image and the postcontrast 3D T1-weighted Dixon water image. Other sequences were also used for identifying tumor location. CCT was used for the evaluation of bone cortex because CT is superior to MR imaging for the detection of bone cortex invasion. When CT and MR image findings were mismatched for bone marrow invasion, the diagnosis obtained on the basis of MR imaging was used.

\section{Statistical Analysis}

For estimating the sensitivity and specificity of both modalities for the detection of skull base invasion, we considered diagnostic confidence scores of $>3$ as positive diagnoses and generated con- tingency tables separately for each site on the skull base. For comparisons of sensitivity and specificity, the McNemar test and generalized estimating equations were used to take into account correlations between multiple sites in the same patient. ${ }^{22}$ The overall diagnostic performance was quantified by the area under the receiver operating characteristic curve using a 5-point scale. Interreader agreement between the independent evaluations of the 2 readers for the scores based on the CCT-alone images and the CCT-plus-BSI images was estimated by weighted $\kappa$ statistics (weight $=2$ ). This was to account for the fact that differences of $>1$ point between the scores of the 2 readers were more important than smaller differences. ${ }^{23}$ Commercial software (STATA, Version 12.1; StataCorp, College Station, Texas) was used. $P<.05$ was considered a significant difference.

\section{RESULTS}

\section{Skull Base Invasion According to the Reference Standard Findings}

All CCT and BSI images generated from the 2-volume datasets were considered of diagnostic image quality. In total, we examined 264 sites ( 44 in the sphenoid body, 44 in the clivus, 88 in the pterygoid process, and 88 in the petrous apex) from 44 patients. Of these 264 sites, skull base invasion was present at 84 sites (31.8\%) according to the reference standard findings. A total of 26 patients $(59.1 \%)$ showed skull base invasion. Bone sclerosis was observed in $21(47.7 \%)$ of the 44 patients, and gross skull base invasion into the bone marrow was observed in 18 (40.9\%) of 44 patients. Skull base invasion was present at 22/44 sphenoid body sites (50.0\%), 20/44 clivus sites (45.5\%), 10/44 right base of the pterygoid process sites $(22.7 \%), 8 / 44$ left base of the pterygoid process sites (18.2\%), 12/44 right petrous bone sites (27.3\%), and 12/44 left petrous bone sites (27.3\%).

\section{Differentiation of Imaging Findings between CCT Alone and CCT-Plus-BSI Imaging}

The results of the image interpretation and diagnostic performance overall and at each site on the skull base for the CCT-alone and CCT-plus-BSI imaging are summarized in Table 2. Among the 84 skull base invasions, 66 (78.6\%) were correctly detected by CCT alone and 18 (21.4\%) produced false-negative findings ( 4 of the sphenoid body, 9 of the clivus, 1 base of the pterygoid process, and 4 of the petrous apex). With use of both CCT and BSI images, however, the number of false-negative findings decreased from 18 to 6 because the BSI images demonstrated iodine enhancement at the skull base without obvious cortical destruction. Figure 1 shows a representative case of a false-negative finding on CCT alone. On CCT images, extensive destruction or sclerosis of the skull base was unclear, but the BSI images clearly demonstrated iodine distribution within soft tissue and bone (both cortex and bone marrow space) continuous with the tumor. Seventy-four true-positive sites on CCT-plus-BSI had an intensity similar to that of the tumor on T2-weighted images. The remaining 4 cases showed hyperintensity on T2-weighted images compared with the primary tumor. Six (7.1\%) false-negative diagnoses of 84 sites remained even after examination of BSI images, due to failure to detect visible iodine enhancement in the bone marrow continuous with the tumor. Among these 6 false-negative diagnoses, 4 
Table 2: Comparison between CCT images alone and CCT-plus-BSI images of skull base invasion ${ }^{\mathrm{a}}$

\begin{tabular}{|c|c|c|c|c|c|c|c|c|c|c|}
\hline Parameter & $T P^{b}$ & $\mathrm{TN}^{\mathrm{b}}$ & $\mathrm{FN}^{\mathrm{b}}$ & $\mathrm{FP}^{\mathrm{b}}$ & $\begin{array}{c}\text { Sensitivity } \\
(\%)\end{array}$ & $P$ Value & $\begin{array}{c}\text { Specificity } \\
(\%)\end{array}$ & $P$ Value & $\begin{array}{l}\text { PPV } \\
\text { (\%) }\end{array}$ & $\begin{array}{l}\text { NPV } \\
(\%)\end{array}$ \\
\hline \multicolumn{11}{|l|}{ All sites } \\
\hline CCT alone & 66 & 155 & 18 & 25 & 79 (68-87) & $.016^{c}$ & $86(80-90)$ & $.010^{c}$ & 73 & 90 \\
\hline CCT-plus-BSI & 78 & 172 & 6 & 8 & 93 (85-97) & & $96(91-98)$ & & 91 & 97 \\
\hline \multicolumn{11}{|l|}{ Sphenoid body } \\
\hline CCT alone & 18 & 14 & 4 & 8 & $82(60-95)$ & .625 & $64(41-83)$ & $.039^{d}$ & 69 & 78 \\
\hline CCT-plus-BSI & 20 & 21 & 2 & 1 & 91 (71-99) & & $95(77-100)$ & & 95 & 91 \\
\hline \multicolumn{11}{|l|}{ Clivus } \\
\hline CCT alone & 11 & 22 & 9 & 2 & $55(32-77)$ & $.031^{\mathrm{d}}$ & $92(73-99)$ & 1.000 & 85 & 71 \\
\hline CCT-plus-BSI & 17 & 22 & 3 & 2 & 85 (62-97) & & 92 (73-99) & & 89 & 88 \\
\hline \multicolumn{11}{|c|}{ Base of the pterygoid process } \\
\hline CCT alone & 17 & 60 & 1 & 10 & $94(73-100)$ & .157 & $86(75-93)$ & $.012^{c}$ & 63 & 98 \\
\hline CCT-plus-BSI & 18 & 68 & 0 & 2 & $100(81-100)$ & & $97(90-100)$ & & 90 & 100 \\
\hline \multicolumn{11}{|l|}{ Petrous apex } \\
\hline CCT alone & 20 & 59 & 4 & 5 & $83(63-95)$ & .221 & $92(83-97)$ & .306 & 80 & 94 \\
\hline CCT-plus-BSI & 23 & 61 & 1 & 3 & $96(79-100)$ & & 95 (87-99) & & 88 & 98 \\
\hline
\end{tabular}

Note:-FN indicates false-negative findings; FP, false-positive findings; NPV, negative predictive value; PPV, positive predictive value; TN, true-negative findings; TP, truepositive findings.

${ }^{a}$ Numbers in parentheses are $95 \%$ confidence intervals.

${ }^{b}$ Data are numbers of findings.

c $P<.05$, according to the generalized estimating equations that accounted for the multiple observations within patients.

${ }^{\mathrm{d}} \mathrm{P}<.05$, as determined with the McNemar test.

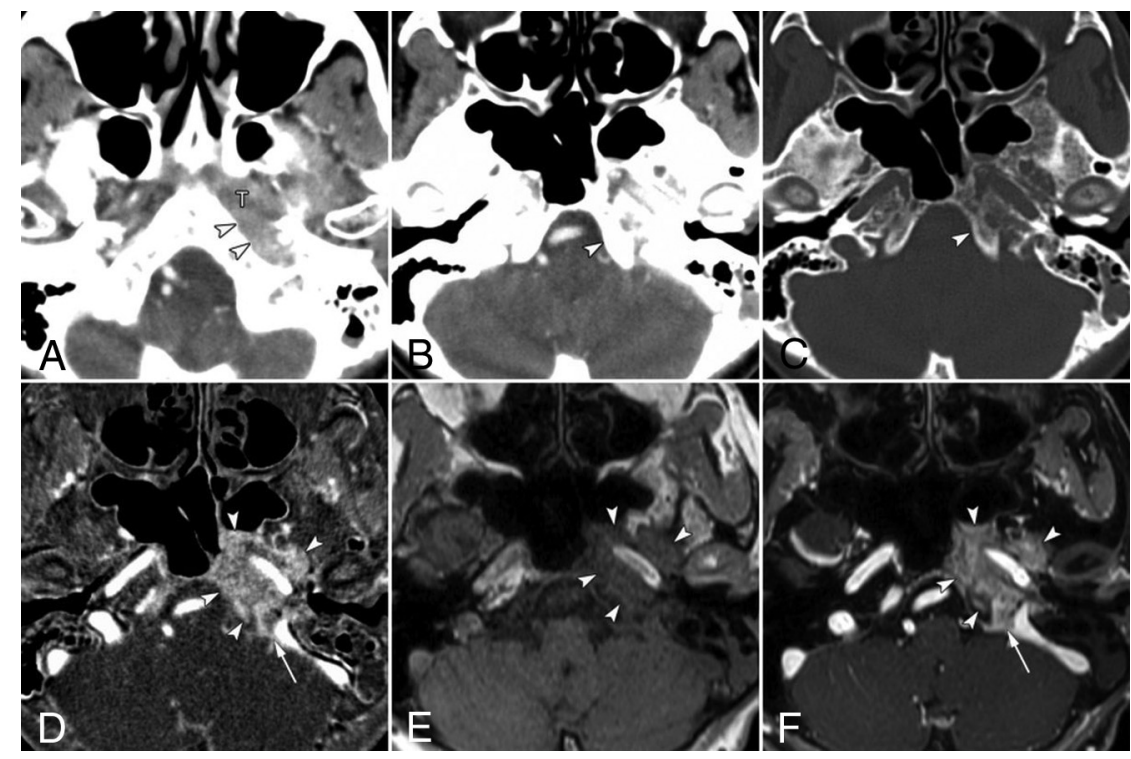

FIG 1. False-negative findings for skull base invasion by conventional CT images alone in a 74year-old woman with nasopharyngeal carcinoma. A, Axial contrast-enhanced CCT image (softtissue window) shows nasopharyngeal tumor (T) spread into the lateral soft tissue around the foramen lacerum (arrowheads). CCT images at the skull base level ( $B$, soft-tissue window; $C$, bone window) show no destruction of the skull base at the clivus (arrowhead). $D$, Bone subtraction iodine image shows remarkable skull base invasion into the bone marrow space such as the clivus, petrous apex, and sphenoid bone (arrowheads) with intracranial extension at the jugular foramen (arrow). A corresponding slice on a T1-weighted image $(E)$ and fat-suppressed T1-weighted image after gadolinium administration $(F)$ show tumor invading the clivus (arrowheads) and spread into the jugular foramen (arrow).

sites in 1 patient were not detected because the tumor showed a weak enhancement with density similar to that of normal bone marrow.

A total of 25 (13.9\%) false-positive findings (8 of the sphenoid body, 2 of the clivus, 10 of the pterygoid process, and 5 of the petrous apex) of 180 sites without skull base invasion were observed on CCT-alone images, whereas 8 (4.4\%) false-positive findings ( 1 sphenoid body, 2 of the clivus, 2 of the pterygoid process, and 3 of the petrous apex) were observed on CCT-plus-
BSI images. The number of sites with false-positive findings decreased from 25 to 8 on CCT-plus-BSI imaging because the BSI images could detect an absence of corresponding enhancement in the sclerotic regions indicated on the CCT images. Figure 2 shows a case with a false-positive finding on CCT alone with sclerotic change. Two of 8 falsepositive sites ( 1 of the clivus and 1 of the petrous apex) seen on CCT-plus-BSI were probably observed due to enhancement of normal red bone marrow in a young patient in her 30s, and 1 site (sphenoid body) was observed due to misidentifying noise as contrast enhancement. The other 5 false-positive findings seen on CCT-plus-BSI were caused by equivocal enhancement in the BSI images and were scored mainly on the basis of erosion or sclerosis observed on the CCT images.

\section{Diagnostic Performance of CCT Alone and CCT-Plus-BSI Imaging}

In an analysis of all sites taken together, the sensitivity of CCT-plus-BSI (93\%; 95\% CI, 85\%-97\%) for the detection of skull base invasion was significantly higher $(P=.02)$ than that of CCT alone (79\%; 95\% CI, 68\%-87\%). If we split the analysis by site, for the clivus the sensitivity of CCT-plus-BSI was significantly higher $(P=.03)(85 \% ; 95 \%$ CI, 62\%-97\%) than that of CCT alone $(55 \%$; $95 \%$ CI, 32\%-77\%). For the other sites, the sensitivity for CCT-plusBSI was higher than that for CCT alone, but none of the differences were significant: sphenoid body ( $P=.63$; $91 \%$ for CCT-plus-BSI versus $82 \%$ for CCT-alone), pterygoid process $(P=.16 ; 100 \%$ versus $94 \%)$, and petrous apex $(P=.22 ; 96 \%$ versus $83 \%)$. 


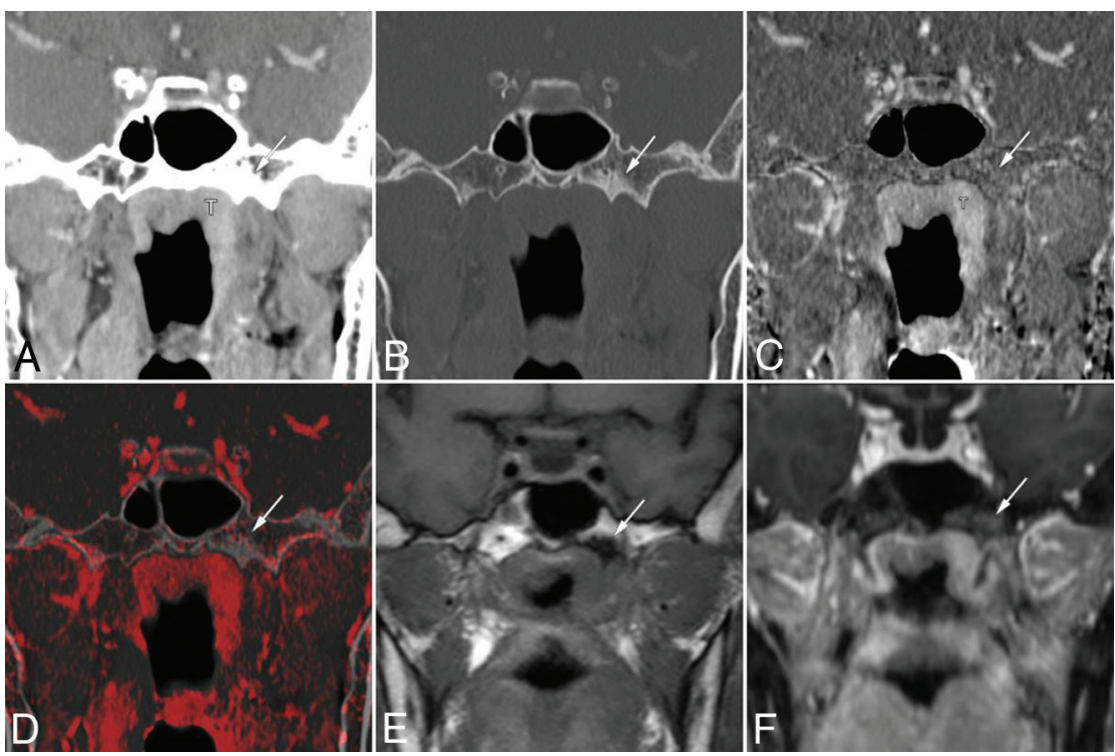

FIG 2. False-positive findings at the base of the left pterygoid process on conventional $C T$ images alone in a 63-year-old woman with nasopharyngeal carcinoma. Coronal contrast-enhanced CCT images ( $A$, soft-tissue window; $B$, bone window) show nasopharyngeal tumor $(T)$ and bone sclerosis at the left base of the pterygoid process (arrow). The case was given a score of 3 based on $C C T$ images alone. Bone subtraction iodine image $(C)$ and color fusion image $(D)$ clearly show no enhancement in the sclerotic area (arrow). E, A corresponding slice on the T1-weighted image shows low signal intensity due to sclerosis (arrow). F, Fat-suppressed Tl-weighted images after gadolinium administration show contrast enhancement of the tumor mass and poor enhancement of the pterygoid process (arrow). This was considered a case of clinical T2 category disease without skull base invasion and was treated by chemoradiotherapy.

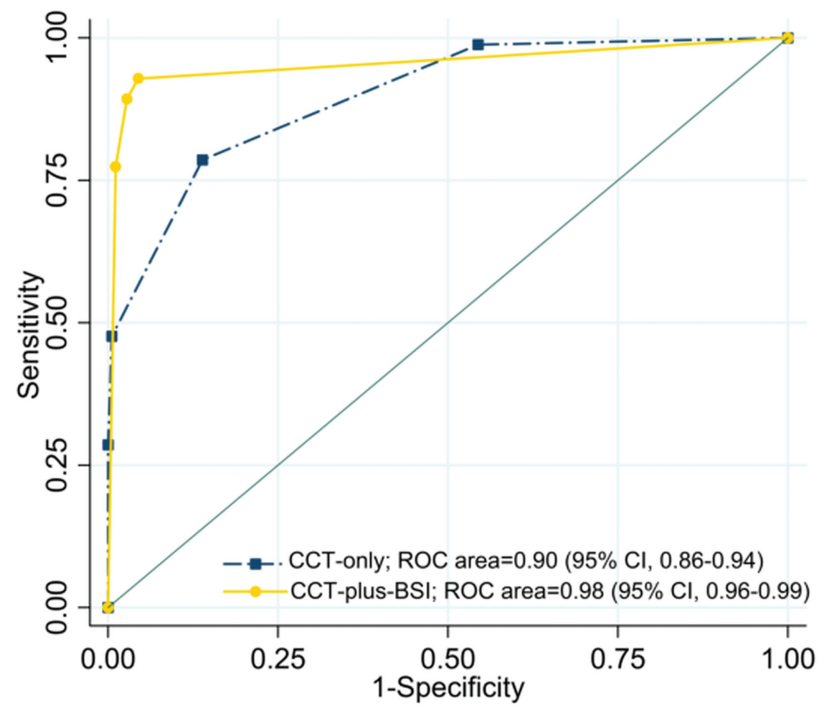

FIG 3. Receiver operating characteristic curves and corresponding areas under the curve for the prediction of skull base invasion. The AUC for CCT-plus-BSI imaging was significantly larger (AUC $=0.98$ $[P<.001])$ than that for CCT imaging alone (AUC $=0.90$ ).

The specificity of CCT-plus-BSI (96\%; 95\% CI, 91\%-98\%) for the detection of skull base invasion was significantly higher $(P=$ $.01)$ than that of CCT alone (86\%; 95\% CI, 80\%-90\%) in the analysis of all sites taken together. When we considered each site separately, the specificity of sphenoid body and pterygoid process detection was significantly higher $(P=.04$ and $P=.012$, respectively) in the case of CCT-plus-BSI (sphenoid body: 95\%; 95\% CI, 77\%-100\%; pterygoid process: $97 \%$; 95\% CI, 90\%-100\%) than that of CCT alone (sphenoid body: 64\%; 95\% CI, 41\%-83\%; pterygoid process: 86\%; 95\% CI, 75\%-93\%). The specificity of CCT-plus-BSI was the same or higher than that of CCT alone at the other sites as well, but none of the differences were significant (clivus: $P=1.00$, $92 \%$ for CCT-plus-BSI versus $92 \%$ for CCT-alone; petrous apex: $P=.31,95 \%$ versus $92 \%$ ).

The receiver operating characteristic curves for skull base invasion and the corresponding AUCs for diagnosis based on CCT-plus-BSI and CCT-alone are shown in Fig 3. The AUC for CCTplus-BSI was significantly larger (AUC = $0.98, P<.001)$ than the AUC for CCT alone $(\mathrm{AUC}=0.90)$.

\section{Interobserver Reproducibility}

The interreader agreement of the 2 readers for CCT alone and for CCT-plus-BSI was assessed with quadratic weighted statistics, with the following results: agreement of 0.82 (95\% CI, 0.53-1.00) and 0.86 (95\% CI, 0.56-1.00), respectively, for the sphenoid body; agreement of 0.84 (95\% CI, 0.55-1.00) and 0.83 (95\% CI, 0.54-1.00), respectively, for the clivus; agreement of 0.85 (95\% CI, 0.57-1.00) and 0.86 (95\% CI, 0.58-1.00), respectively, for the right base of the pterygoid process; agreement of 0.82 (95\% CI, 0.55-1.00) and 0.81 (95\% CI, 0.53-1.00), respectively, for the left base of the pterygoid process; agreement of 0.81 (95\% CI, 0.52-1.00) and 0.89 (95\% CI, 0.59-1.00), respectively, for the right petrous apex; agreement of 0.85 (95\% CI, 0.57-1.00) and 0.86 (95\% CI, 0.58-1.00), respectively, for the left petrous apex.

\section{DISCUSSION}

The results of our study demonstrate that the combined analysis of CCT and BSI images showed a better diagnostic performance than the analysis of CCT images alone in the evaluation of skull base invasion in patients with NPC. These results suggest that CCT-plus-BSI images potentially allow an assessment closely resembling that provided by combining CT and contrast-enhanced MR imaging. High sensitivity could be achieved because CCTplus-BSI improves the detection of tumor spread within the skull base (cortical bone and bone marrow), especially in the clivus. Another factor contributing to the improved specificity was that CCT-plus-BSI reduced the overestimation caused by sclerotic parts such as the sphenoid body and pterygoid process, which may reflect the bone response to tumor proximity. The differences in the diagnostic performance of each site may be due to the amount of bone marrow, the thickness of the bone affected by pneumatization, and the frequency of sclerosis. These results may be particularly useful for improving the accuracy for radiation therapy treatment planning and primary tumor staging. Further- 
more, CCT-plus-BSI imaging offers a benefit for patients with contraindications to MR imaging due to metallic foreign bodies, MR imaging-unsafe pacemaker devices, or claustrophobia.

CT is an excellent technique for the evaluation of bone details, particularly for the evaluation of cortical bone invasion. However, it is still challenging to assess tumor extent in the bone marrow using CCT imaging without contrast-enhanced MR imaging because of low contrast resolution and beam-hardening artifacts produced by the bone cortex. MR imaging is superior to CT in the detection of skull base involvement. ${ }^{8-11}$ Several studies have used MR imaging to examine abnormalities of the skull base in patients with NPC. ${ }^{17-21,24} \mathrm{Lu}$ et $\mathrm{al}^{20}$ reported worse prognosis when $\geq 2$ sites of skull base invasion were found on MR imaging. Other groups found that the prognosis varied depending on the sites of skull base invasion. ${ }^{17,21,24}$ Therefore, from the perspective of prognosis, comprehensive assessment is needed, including the sites and spatial extent within the skull base. BSI images have the potential to detect enhancement in the bone marrow and may complement CCT in assessing bone invasion.

Shatzkes et $\mathrm{al}^{25}$ reported that sclerotic findings in the pterygoid process are detected on CT in 60\% of cases of untreated NPC. These findings may indicate present or imminent skull base invasion and may be used for tumor staging and the formulation of intensity-modulated radiotherapy fields. These sclerotic changes may be a sensitive indicator of skull base invasion, but they are not specific because sclerosis can also be caused by inflammatory or reactive changes. ${ }^{26,27}$ Recently, there have been several reports of the use of dual-energy CT for the diagnosis of bone marrow edema in patients with bone fractures ${ }^{28-30}$ and for the detection of bone marrow involvement in patients with multiple myeloma. ${ }^{31}$ However, with current dual-energy CT systems and material decomposition algorithm, dual-energy CT technology cannot clearly distinguish bone from iodinated contrast. ${ }^{32,33}$ Thus, calcium (bone) remains visible on the iodine map. BSI images generated by area detector CT allow bone removal (both cortical and trabecular) without affecting the visualization of contrast enhancement in a nonlytic bone background. ${ }^{12-14}$ BSI imaging may be helpful for the diagnosis of bone invasion with or without erosive changes in cortical bone.

There are limitations to our study. The first is the absence of histopathologic verification of the findings of skull base invasion because the difficulty in accessing the skull base precludes surgical confirmation and almost all patients were treated with chemoradiotherapy. According to a study of MR imaging findings for laryngeal cartilage invasion, contrast enhancement is also caused by reactive inflammation, edema, and fibrosis in close proximity to the tumor. ${ }^{34}$ Therefore, there is no clear consensus on whether the contrast enhancement on BSI images was caused by tumor invasion or inflammation. These issues need to be examined at other primary sites such as the mandibular bone or maxillary bone in future studies. The second potential limitation is that skull base invasion was assessed only visually, without quantitative measurement of iodine concentrations. Therefore, potentially confounding effects of interreader error, including display settings, may be present. Moreover, the degree by which tumor and bone marrow enhancement influence the quality and diagnostic per- formance of BSI images remains unclear and needs to be evaluated in future studies.

\section{CONCLUSIONS}

The combined analysis of CCT and BSI images generated from 320 -row area detector CT has the potential to improve diagnostic performance in the evaluation of skull base invasion by NPC. BSI images can provide additional contrast resolution for bone assessment. Although there is no direct comparison between the underlying tumor histopathology and the results of BSI imaging, CCTplus-BSI imaging may be useful for accurate staging of the tumor and may be helpful for patients with contraindications for MR imaging.

Disclosures: Takashi Hiyama—RELATED: Consulting Fee or Honorarium: Canon Medical Systems. * Hirofumi Kuno-RELATED: Grant: Grant-in-Aid for Young Scientists (B) KAKEN (No. 26861033); Consulting Fee or Honorarium: Canon Medical Systems*; UNRELATED: Grants/Grants Pending: Grant-in-Aid for Young Scientists KAKEN (No. 18K15573). So Tsushima—UNRELATED: Employment: Canon Medical Systems. Osamu Sakai-UNRELATED: Consultancy: Boston Imaging Core Lab. Masahiko Kusumoto-RELATED: Consulting Fee or Honorarium: Canon Medical Systems.* Tatsushi Kobayashi-RELATED: Consulting Fee or Honorarium: Canon Medical Systems*; UNRELATED: Grants/Grants Pending: Japan Agency for Medical Research and Development, Grant No. 17ck0106343h0001. * ${ }^{*}$ Money paid to the institution.

\section{REFERENCES}

1. Amin MB, Edge SB, Greene FL, et al. AJCC Cancer Staging Manual. 8th ed. New York: Springer-Verlag; 2017

2. Peng G, Wang T, Yang KY, et al. A prospective, randomized study comparing outcomes and toxicities of intensity-modulated radiotherapy vs. conventional two-dimensional radiotherapy for the treatment of nasopharyngeal carcinoma. Radiother Oncol 2012;104: 286-93 CrossRef Medline

3. Lee AW, Ng WT, Chan LL, et al. Evolution of treatment for nasopharyngeal cancer: success and setback in the intensity-modulated radiotherapy era. Radiother Oncol 2014;110:377-84 CrossRef Medline

4. Mukherji SK, Pillsbury HR, Castillo M. Imaging squamous cell carcinomas of the upper aerodigestive tract: what clinicians need to know. Radiology 1997;205:629-46 CrossRef Medline

5. Dubrulle F, Souillard R, Hermans R. Extension patterns of nasopharyngeal carcinoma. Eur Radiol 2007;17:2622-30 CrossRef Medline

6. King AD, Bhatia KS. Magnetic resonance imaging staging of nasopharyngeal carcinoma in the head and neck. World J Radiol 2010;2: 159-65 CrossRef Medline

7. Abdel Razek AA, King A. MRI and CT of nasopharyngeal carcinoma. AJR Am J Roentgenol 2012;198:11-18 CrossRef Medline

8. Chong VF, Fan YF. Skull base erosion in nasopharyngeal carcinoma: detection by CT and MRI. Clin Radiol 1996;51:625-31 CrossRef Medline

9. Zhang SX, Han PH, Zhang GQ, et al. Comparison of SPECT/CT, MRI and CT in diagnosis of skull base bone invasion in nasopharyngeal carcinoma. Biomed Mater Eng 2014;24:1117-24 CrossRef Medline

10. Ng SH, Chang TC, Ko SF, et al. Nasopharyngeal carcinoma: MRI and CT assessment. Neuroradiology 1997;39:741-46 CrossRef Medline

11. Liao XB, Mao YP, Liu LZ, et al. How does magnetic resonance imaging influence staging according to AJCC staging system for nasopharyngeal carcinoma compared with computed tomography? Int J Radiat Oncol Biol Phys 2008;72:1368-77 CrossRef Medline

12. Gondim Teixeira PA, Hossu G, Lecocq S, et al. Bone marrow edema pattern identification in patients with lytic bone lesions using digital subtraction angiography-like bone subtraction on large-area detector computed tomography. Invest Radiol 2014;49:156-64 CrossRef Medline

13. Gondim Teixeira PA, Gervaise A, Louis M, et al. Musculoskeletal wide detector CT: principles, techniques and applications in clini- 
cal practice and research. Eur J Radiol 2015;84:892-900 CrossRef Medline

14. Kuno H, Sekiya K, Chapman MN, et al. Miscellaneous and emerging applications of dual-energy computed tomography for the evaluation of intracranial pathology. Neuroimaging Clin N Am 2017;27: 411-27 CrossRef Medline

15. Edge SE, Byrd DR, Compton CC, et al, eds. AJCC Cancer Staging Manual. 7th ed. New York: Springer-Verlag; 2009

16. Crum WR, Hill DL, Hawkes DJ. Information theoretic similarity measures in non-rigid registration. Inf Process Med Imaging 2003;18: 378-87 Medline

17. Chen L, Liu LZ, Mao YP, et al. Grading of MRI-detected skull-base invasion in nasopharyngeal carcinoma and its prognostic value. Head Neck 2011;33:1309-14 CrossRef Medline

18. Li YZ, Cai PQ, Xie CM, et al. Nasopharyngeal cancer: impact of skull base invasion on patients prognosis and its potential implications on TNM staging. Eur J Radiol 2013;82:e107-11 CrossRef Medline

19. Nishioka T, Shirato H, Kagei K, et al. Skull-base invasion of nasopharyngeal carcinoma: magnetic resonance imaging findings and therapeutic implications. Int J Radiat Oncol Biol Phys 2000;47:395400 CrossRef Medline

20. Lu JC, Wei Q, Zhang YQ, et al. Influence of MRI abnormality in skull base bone on prognosis of nasopharyngeal carcinoma. Cancer Radiother 2004;8:230-33 CrossRef Medline

21. Wang $\mathrm{H}$, Zhang $\mathrm{ZQ}$, Huang $\mathrm{LL}$, et al. MR imaging prediction of local control of nasopharyngeal carcinoma treated with radiation therapy and chemotherapy. Br J Radiol 2014;87:20130657 CrossRef Medline

22. Genders TS, Spronk S, Stijnen T, et al. Methods for calculating sensitivity and specificity of clustered data: a tutorial. Radiology 2012; 265:910-16 CrossRef Medline

23. Sim J, Wright CC. The kappa statistic in reliability studies: use, interpretation, and sample size requirements. Phys Ther 2005;85: 257-68 Medline

24. Roh JL, Sung MW, Kim KH, et al. Nasopharyngeal carcinoma with skull base invasion: a necessity of staging subdivision. Am J Otolaryngol 2004;25:26-32 CrossRef Medline
25. Shatzkes DR, Meltzer DE, Lee JA, et al. Sclerosis of the pterygoid process in untreated patients with nasopharyngeal carcinoma. $R a$ diology 2006;239:181-86 CrossRef Medline

26. Becker M, Zbären P, Delavelle J, et al. Neoplastic invasion of the laryngeal cartilage: reassessment of criteria for diagnosis at CT. Radiology 1997;203:521-32 CrossRef Medline

27. Muñoz A, Ramos A, Ferrando J, et al. Laryngeal carcinoma: sclerotic appearance of the cricoid and arytenoid cartilage-CT-pathologic correlation. Radiology 1993;189:433-37 CrossRef Medline

28. Kaup M, Wichmann JL, Scholtz JE, et al. Dual-energy CT-based display of bone marrow edema in osteoporotic vertebral compression fractures: impact on diagnostic accuracy of radiologists with varying levels of experience in correlation to MR imaging. Radiology 2016;280:510-19 CrossRef Medline

29. Pache G, Krauss B, Strohm P, et al. Dual-energy CT virtual noncalcium technique: detecting posttraumatic bone marrow lesionsfeasibility study. Radiology 2010;256:617-24 CrossRef Medline

30. Kellock TT, Nicolaou S, Kim SSY, et al. Detection of bone marrow edema in nondisplaced hip fractures: utility of a virtual noncalcium dual-energy CT application. Radiology 2017;284:798-805 CrossRef Medline

31. Thomas C, Schabel C, Krauss B, et al. Dual-energy CT: virtual calcium subtraction for assessment of bone marrow involvement of the spine in multiple myeloma. AJR Am J Roentgenol 2015;204: W324-31 CrossRef Medline

32. Gupta R, Phan CM, Leidecker C, et al. Evaluation of dual-energy CT for differentiating intracerebral hemorrhage from iodinated contrast material staining. Radiology 2010;257:205-11 CrossRef Medline

33. Kuno H, Onaya H, Iwata R, et al. Evaluation of cartilage invasion by laryngeal and hypopharyngeal squamous cell carcinoma with dualenergy CT. Radiology 2012;265:488-96 CrossRef Medline

34. Becker M, Zbären P, Casselman JW, et al. Neoplastic invasion of laryngeal cartilage: reassessment of criteria for diagnosis at MR imaging. Radiology 2008;249:551-59 CrossRef Medline 